\title{
Anatomic Foveal Reconstruction of the Triangular Fibrocartilage Complex With a Tendon Graft
}

\author{
Gregory I. Bain, MBBS, FRACS, FA (Orth) A, PhD,*t+ \\ Duncan McGuire, MBBCH, FCS (Orth), MMed, * + Yu Chao Lee, MBBS, *† \\ Kevin Eng, MBBS, FRACS, * $\dagger$ and Matthias Zumstein, MD§
}

\begin{abstract}
An acute injury to the triangular fibrocartilage complex (TFCC) with avulsion of the foveal attachment can produce distal radioulnar joint (DRUJ) instability. The avulsed TFCC is translated distally so the footprint will be bathed in synovial fluid from the DRUJ and will become covered in synovitis. If the TFCC fails to heal to the footprint, then persistent instability can occur. The authors describe a surgical technique indicated for the treatment of persistent instability of the DRUJ due to foveal detachment of the TFCC. The procedure utilizes a loop of palmaris longus tendon graft passed through the ulnar aspect of the TFCC and into an osseous tunnel in the distal ulna to reconstruct the foveal attachment. This technique provides stability of the distal ulna to the radius and carpus. We recommend this procedure for chronic instability of the DRUJ due to TFCC avulsion, but recommend that suture repair remain the treatment of choice for acute instability. An arthroscopic assessment includes the trampoline test, hook test, and reverse hook test. DRUJ ballottement under arthroscopic vision details the direction of instability, the functional tear pattern, and unmasks concealed tears. If the reverse hook test demonstrates a functional instability between the TFCC and the radius, then a foveal reconstruction is contraindicated, and a reconstruction that stabilizes the radial and ulnar aspects of the TFCC is required. The foveal reconstruction technique has the advantage of providing a robust anatomically based reconstruction of the TFCC to the fovea, which stabilizes the DRUJ and the ulnocarpal sag.
\end{abstract}

Key Words: triangular fibrocartilage complex, foveal tear, DRUJ instability, wrist arthroscopy, TFCC repair

(Tech Hand Surg 2014;18: 92-97)

\section{HISTORICAL PERSPECTIVE}

The triangular fibrocartilage complex (TFCC) is a complex 3-dimensional structure made up of the proximal triangular ligament or the radioulnar ligaments (RUL), the distal hammock structure, and the ulnar collateral ligaments. ${ }^{1,2}$ The distal hammock structure and the ulnar collateral ligaments are distal components, inserting into the ulnar styloid. The palmar and dorsal portions of the RUL originate from the distal margin of the sigmoid notch and converge to attach to the ulna. As the ligaments extend ulnarly, they divide into a distal component that attaches to the ulnar styloid and a proximal component that attaches to the fovea. ${ }^{3-6}$ The articular disk

From the *Department of Orthopaedic Surgery, Modbury Public Hospital; $\dagger$ Department of Orthopaedics and Trauma, Royal Adelaide Hospital; †Department of Anatomy, University of Adelaide, Adelaide, Australia; and $\S$ Upper Extremity Unit, Department of Orthopaedic Surgery Traumatology, University of Bern, Inselspital, Bern, Switzerland.

Conflicts of Interest and Source of Funding: The authors report no conflicts of interest and no source of funding.

Address correspondence and reprint requests to Gregory I. Bain, MBBS, FRACS, FA (Orth) A, PhD, 196 Melbourne Street, North Adelaide 5006, SA, Australia. E-mail: greg@gregbain.com.au.

Copyright (C) 2014 by Lippincott Williams \& Wilkins extends from the ulnar edge of the lunate fossa at the distal rim of the sigmoid notch and blends peripherally with the RUL.

The foveal insertion of the TFCC contributes more to DRUJ stability than the styloid insertion because of its closer relationship to the rotational axis of the forearm. ${ }^{4}$ In fractures of the ulnar styloid with disruption of the distal components of TFCC, patients may not experience DRUJ instability due to an intact foveal attachment. ${ }^{7}$ However, disruption of the TFCC at its foveal attachment can result in DRUJ instability. ${ }^{3,5}$

Palmer $^{8}$ proposed a classification system for TFCC tears that divided them into traumatic and degenerative. Class I B tears are traumatic ulnar-sided peripheral tears that are more amendable to repair. ${ }^{9}$ Atzei and Luchetti $^{2}$ recently subclassified the Palmer 1B tear into a distal component, proximal component, or both (complete) to guide treatment. Anatomic TFCC repair into the fovea is recommended for proximal or complete tears. $^{2}$

Conventional repair techniques for peripheral TFCC tears involve repair to the dorsal or ulnar joint capsule. They do not reconstitute the foveal origin, and instability may persist. Estrella et $\mathrm{al}^{9}$ reported results of a capsular repair of peripheral TFCC tears with unsatisfactory results in $26 \%$, of which $45 \%$ were related to persistent DRUJ instability. Normally, the palmar and dorsal limbs are isometric in length. A nonisometric repair can lead to failure, or a loss of forearm rotation. ${ }^{5}$

Most current techniques described involve suture repair of the TFCC into the fovea. ${ }^{1,5,10}$ However, persistent instability of the DRUJ may persist, resulting in pain and patient dissatisfaction. ${ }^{1,5,11}$ In an attempt to improve these results we present our foveal reconstruction technique using a palmaris longus graft. Adapted from the modified Nakamura technique, the procedure uses the palmaris longus tendon to secure the TFCC into the fovea through an osseous tunnel. Our technique reconstructs the TFCC into the fovea, which offers a theoretical advantage over a suture repair, of a more stable reconstruction after osseous-tendon integration in the tunnel. It stabilises the DRUJ and the ulnar-carpal sling.

The normal TFCC has 3 main attachments: the fovea of the ulna, the radius, and the carpus. It is the authors' opinion that the pathoanatomic reason for the persistent instability with a foveal tear is that the entire TFCC is translated distally because of the intact attachment to the carpus and radius. The foveal footprint becomes bathed in synovial fluid from the DRUJ and becomes covered in synovitis. This may prevent the TFCC healing to the fovea, and persistent DRUJ instability can occur. The authors believe that some of the published failures occur when (a) the surgeon performs a peripheral repair and fails to address the foveal attachment, or (b) performs a foveal repair with a suture or suture anchor but fails to keep the TFCC on its native footprint, so that it does not heal. ${ }^{5}$ The foveal reconstruction described in this paper is aimed at avoiding these 2 scenarios. 


\section{INDICATIONS/CONTRAINDATIONS}

\section{Clinical Assessment}

There is often a history of a fall or rotational injury. The proposed mechanism that causes a foveal tear is hyper radial extension of the wrist. ${ }^{4}$ The patient may describe clicking or pain with activities that involve supination or pronation. Patients may report weakness, especially when manipulating objects.

Examination of the wrist reveals the "foveal sign," which is point tenderness over the ulnar capsule located volar to the extensor carpi ulnaris tendon. ${ }^{12}$ Rotation of the forearm may produce clicking or crepitation of the DRUJ. The ballottement test evaluates DRUJ stability. This is performed by passively translating the ulna in an anteroposterior direction, with the forearm in neutral, full supination, and full pronation. This is compared with the contralateral side, and abnormal translation suggests a complete TFCC tear. ${ }^{2,13}$

Although it is commonly considered that dorsal instability is much more common, it is the authors' experience that volar instability is often overlooked. Volar instability is best assessed in supination, where there is an abnormal prominence of the ulnar head on the volar aspect of the wrist. Because of the flexor tendons, this prominence is not so obvious. On the dorsal aspect of the wrist, there will be an obvious dimple, because of the fact there is no ulnar head creating the usual prominence. When the clinician examines the patient with volar instability, the wrists are usually placed on the examination table. In supination, the volar prominence is easily overlooked and the dorsal dimple is only seen if the examiner looks directly at the dorsal wrist in full supination.

Plain radiographs should be performed looking for an ulnar styloid fracture and DRUJ incongruity. Magnetic resonance imaging can be used to identify the peripheral TFCC tear. ${ }^{14}$ Magnetic resonance imaging arthrogram improves the accuracy of diagnosing a foveal tear; however, there are some cases where there is no leakage of contrast, as the entire TFCC has been avulsed from the fovea. ${ }^{2}$ We often perform a DRUJ arthrogram (instead of radiocarpal), as this will confirm the tear of the foveal attachment of the TFCC (Figs. 1A, B).

\section{Treatment Options}

The DRUJ may be stabilized by intra-articular or extraarticular techniques. Intra-articular techniques include repair or reconstruction of the TFCC. Extra-articular techniques include ulnar-shortening osteotomy and sigmoid notch osteotomy.

\section{Repair}

If the patient has an acute instability, with a repairable TFCC, then a suture repair is all that is required. For patients with a delayed presentation of pain but no instability, the authors would recommend a debridement or repair of the TFCC and not perform a reconstruction.

\section{Reconstruction}

An ulnar-sided foveal TFCC reconstruction can be performed in chronic cases, wherein the TFCC is still attached to the distal radius and the carpus. If there is a small radial-sided tear of the TFCC, but the stability between the TFCC and the distal radius is maintained, then we would still proceed using this technique. A radial-sided instability of the TFCC is identified by probing the tear, utilizing the arthroscopic drawer test, and performing a "reverse hook test," wherein a hooked probe is used to translate the TFCC in the ulnar (reverse) direction. This assessment is important because if there is an extensive or complex tear of the TFCC, then our technique of a foveal reconstruction is contraindicated. If the reverse hook test is positive, then there is no functional stability between the TFCC and the radius. In this case a reconstruction that stabilizes the radial and ulnar aspects of the TFCC, as described by Mansat and colleagues, and Adams and Berger, would be our preferred technique. ${ }^{15,16}$

\section{Osteotomy}

If the patient has changes consistent with ulnocarpal impaction, such as a tear of the TFCC associated with chondral changes on the lunate or triquetrum, then an ulna shortening osteotomy would be the preferred option. By shortening the ulna, this will provide a degree of increased stability, as it will tension the ulnar-carpal ligaments. ${ }^{17,18}$

The DRUJ may also be unstable because of an abnormality of the sigmoid notch. If there is a distal radius malunion, then a distal radius osteotomy will realign the notch and restore stability. A malunion of a fracture extending into the sigmoid notch could be considered for a sigmoid notch osteotomy. ${ }^{19,20}$ If the sigmoid notch is abnormal flat, then a sigmoid notch osteoplasty may provide stability to the ulna head $^{19}$ (Fig. 2).

\section{SURGICAL TECHNIQUE}

\section{Set-up}

The patient is given a general anesthetic, the arm exsanguinated, tourniquet inflated to $250 \mathrm{mmHg}$, and the hand suspended in Chinese finger traps from a shoulder arthroscopy
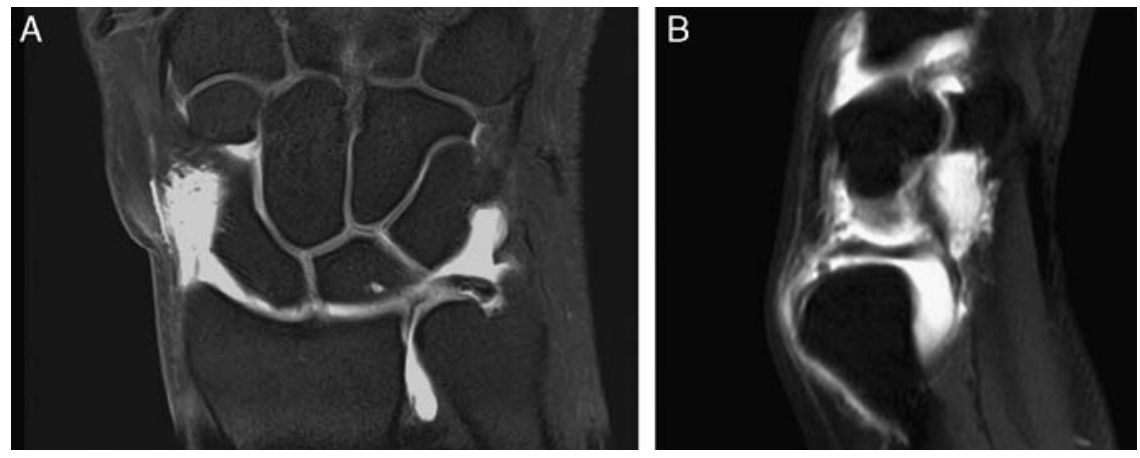

FIGURE 1. A and B, Coronal and sagittal images of a magnetic resonance imaging arthrogram, demonstrating a foveal avulsion of the triangular fibrocartilage complex (TFCC) with dorsal subluxation of the ulnar head. 


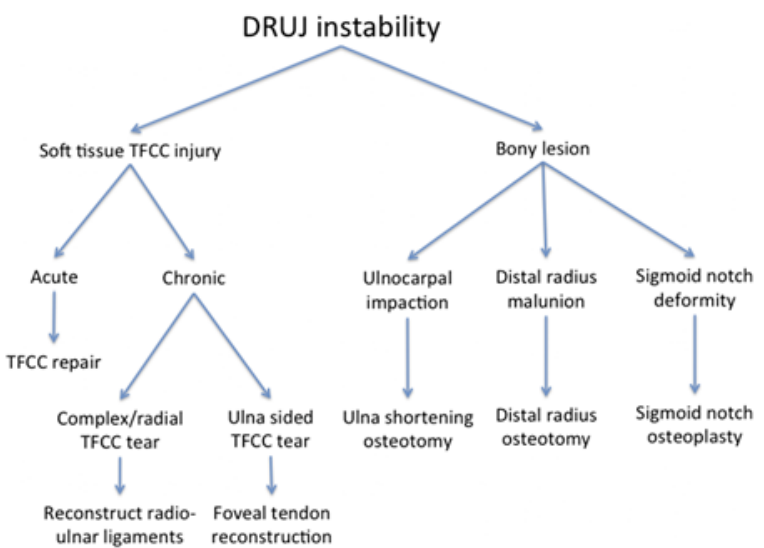

FIGURE 2. Treatment algorithm for the management of distal radioulnar joint (DRUJ) instability. full color

frame. ${ }^{21}$ The stability of the DRUJ is assessed, and the direction of instability is determined.

\section{Arthroscopic Assessment}

A standard wrist arthroscopy is performed. The TFCC is examined to obtain a detailed understanding of the tear configuration. Other structures in the wrist that could be a

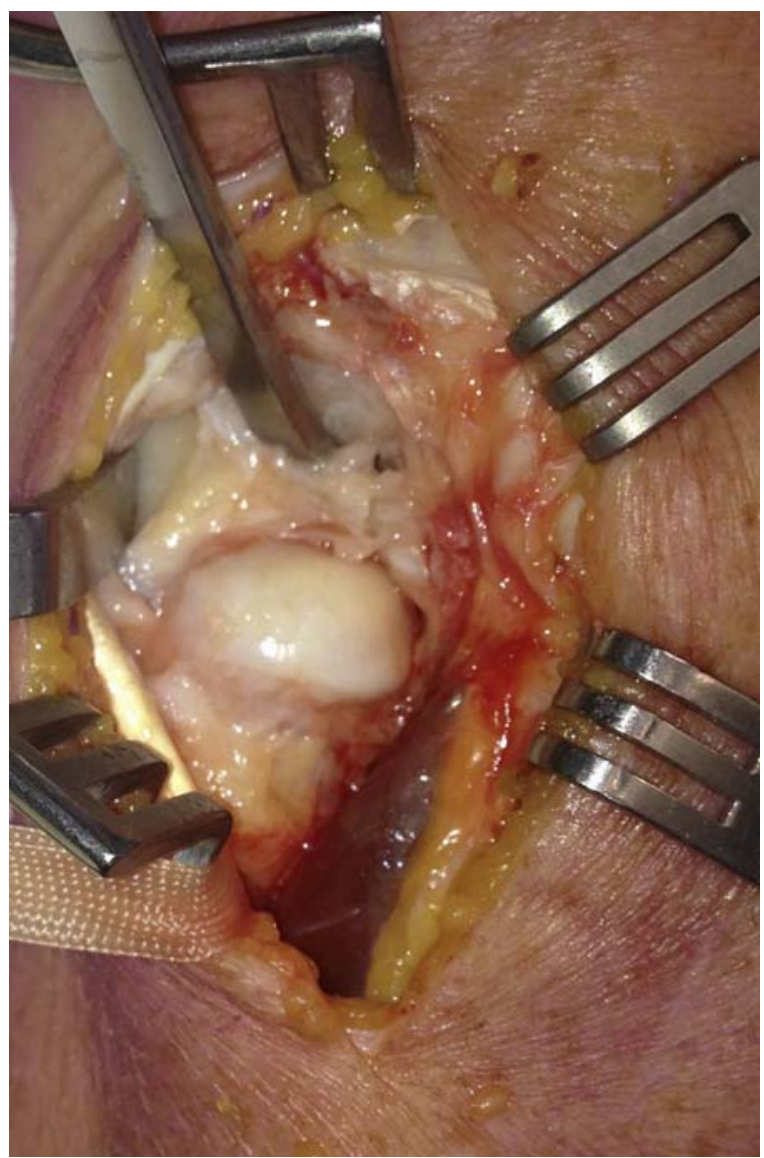

FIGURE 3. Dorsal approach to the distal radioulnar joint (DRUJ) through the bed of the fifth extensor compartment. The instrument is placed in the ulnocarpal joint with the triangular fibrocartilage complex (TFCC) below it. full color

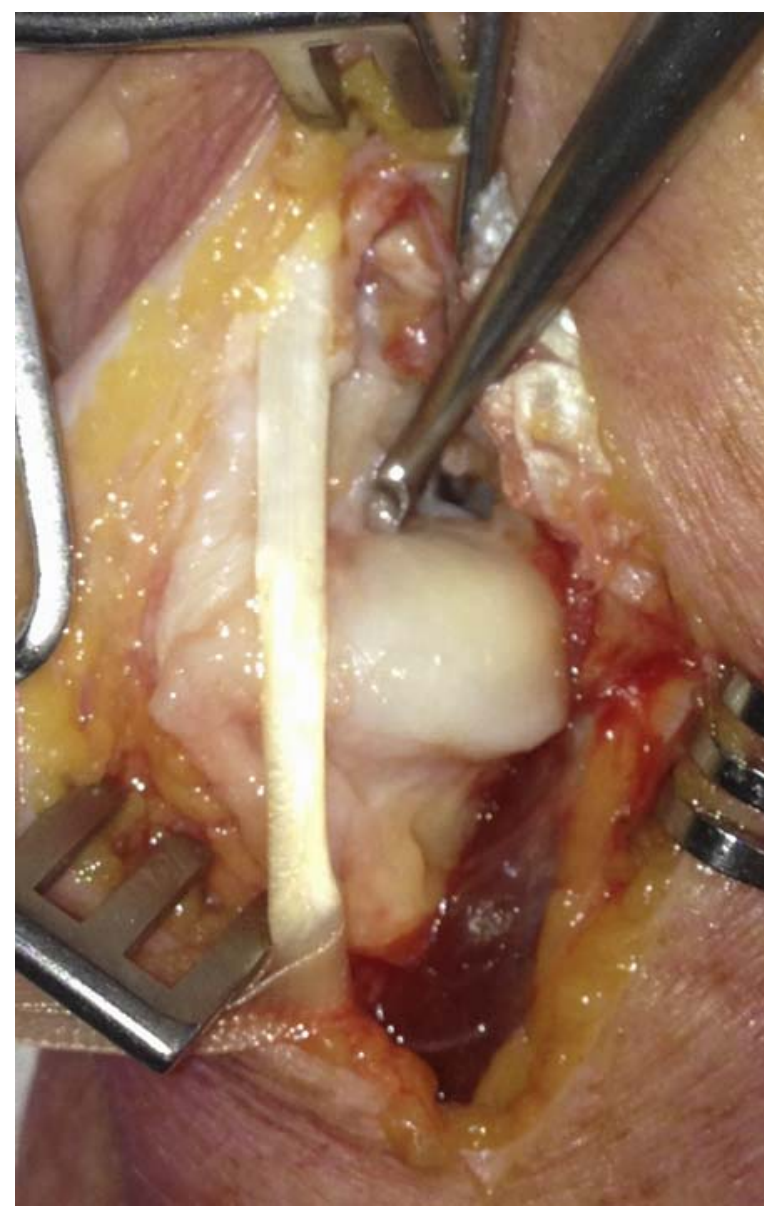

FIGURE 4. The fovea is visualized and debrided with either a curette or motorized resector. full color

source of pain should be assessed, particularly synovitis, tears of the lunotriquetral ligament, and chondral defects. DRUJ arthroscopy can be performed to directly visualize the foveal attachment of the TFCC. This is easier to perform with a foveal disruption, as it creates more space. ${ }^{1}$

\section{Exposure}

A dorsal open approach using the fifth extensor compartment is used as it provides good exposure of the TFCC and fovea. The dorsal branch of the ulnar nerve should be protected. The roof of the fifth extensor compartment is released, and the EDM tendon is retracted. The floor of the fifth compartment is incised and the dorsal capsule is reflected ulnarly, to expose the proximal and distal aspects of the TFCC (Fig. 3).

\section{Distal Ulna Preparation}

A blunt levered retractor can be used to elevate the unstable ulnar head out of the wound. The foveal footprint is debrided with either a motorized resector or a curette (Fig. 4).

Under direct vision, a guide wire is advanced from the fovea to exit the subcutaneous border of the ulna 1.5 to $2 \mathrm{~cm}$ proximal to the tip of the ulnar styloid. A cannulated drill is advanced over the guide wire and the surrounding tissues are protected with a drill guide (Fig. 5). The size of the drill hole is dependent upon the size of the palmaris longus graft when folded and doubled up (usually a 3.5 or $4 \mathrm{~mm}$ drill). 


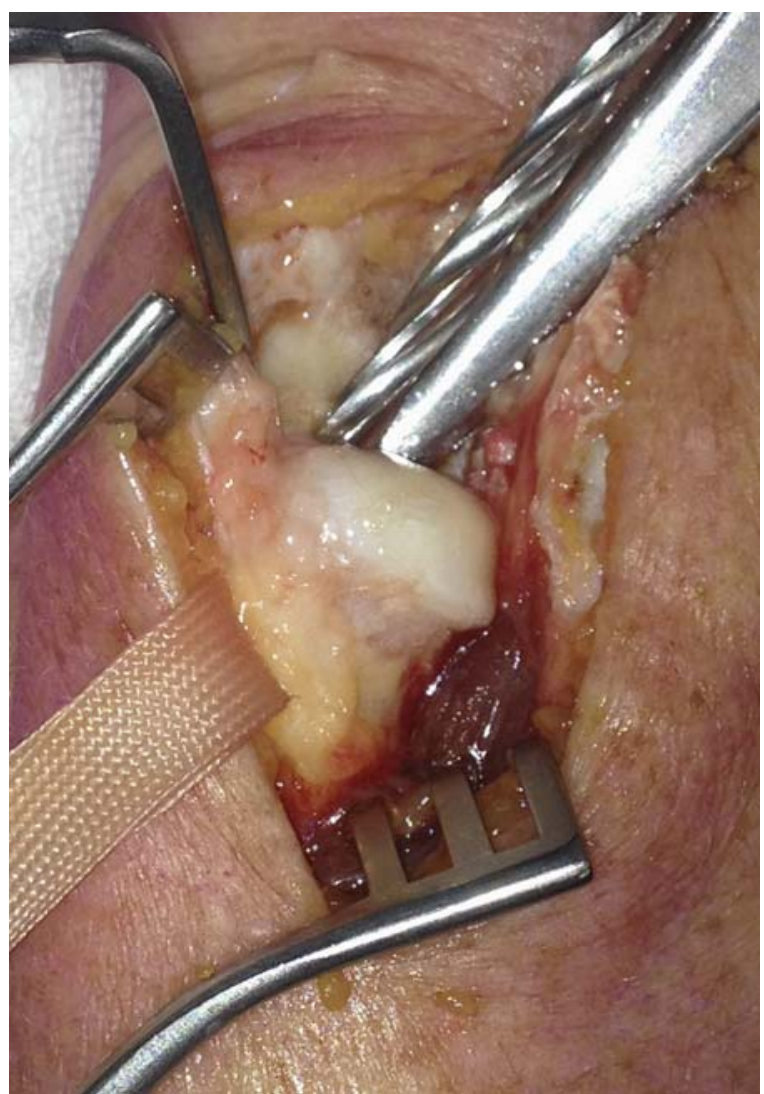

FIGURE 5. A wire is advanced from the fovea to the subcutaneous border of the ulna under direct vision. A cannulated drill is then advanced over the wire. full color

\section{Placement of the Palmaris Longus Tendon Graft}

The palmaris longus tendon is harvested from the volar aspect of the wrist and grasping sutures are placed into each end of the tendon. Both ends of the sutures and tendon are passed through the TFCC from distal to proximal and then through the ulnar drill hole using a suture loop or suture passer (Fig. 6). Once the sutures and graft are tensioned, the TFCC is pulled down onto the foveal footprint, thereby stabilizing the DRUJ. The dorsal capsule is plicated, which adds further stability to the DRU (Fig. 7).

\section{Stabilization of the Tendon Graft to the Ulna}

Our preferred technique is to use a tensionable anchor inserted into the subcutaneous border of the ulna, proximal to the drill hole. A tensionable anchor is a knotless anchor where sutures are first loaded into the anchor, the anchor is then deployed, and the sutures are then tensioned in a controlled manner.

We have previously used interference fit screws and Endobuttons. The interference fit screws are technically the simplest; however, we are concerned about the risk of fracture of the distal ulna. The Endobutton migrates distally because of the obliquity of the ulnar hole and is palpable on the subcutaneous border of the ulna.

The patient is placed into an above-elbow cast postoperatively (Figs. 8A, B, 9A, B).

\section{REHABILITATION}

The above-elbow cast is removed after 4 weeks, and the patient is provided with a removable wrist splint for further 4 weeks.

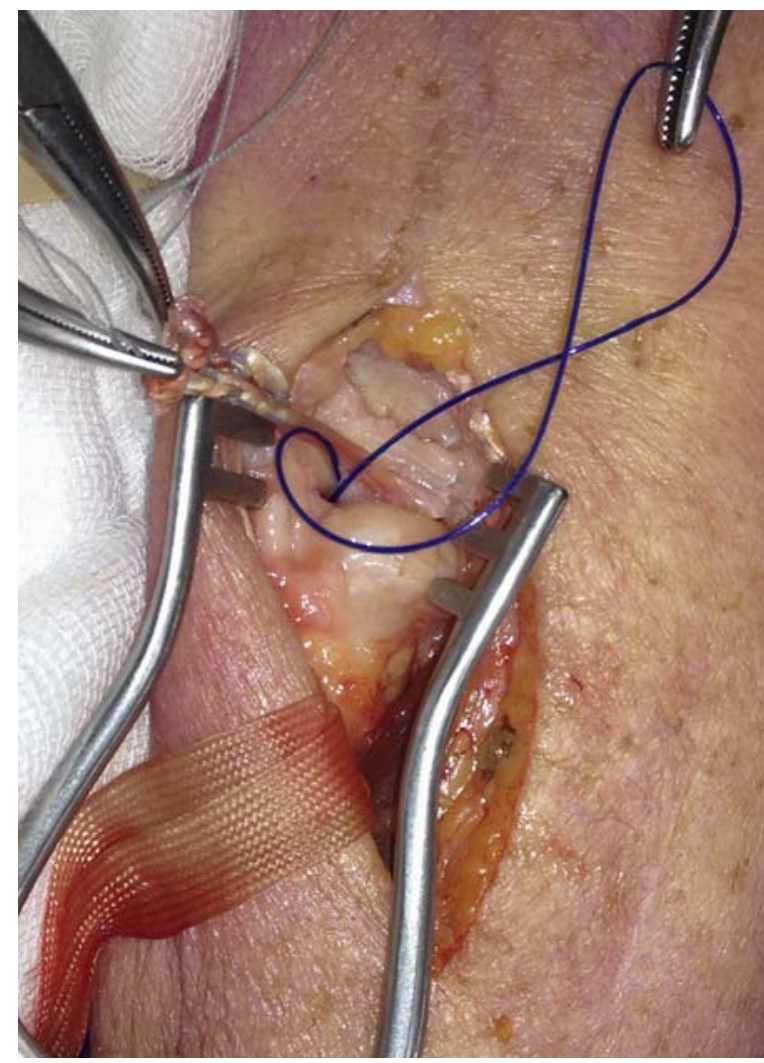

FIGURE 6. The palmaris longus graft has been passed through the triangular fibrocartilage complex (TFCC). A loop of suture is passed through the ulna drill hole and is used to retrieve the suture ends, after which the graft is pulled down into the bone tunnel. $\frac{\text { full color }}{0 \mathrm{nline}}$

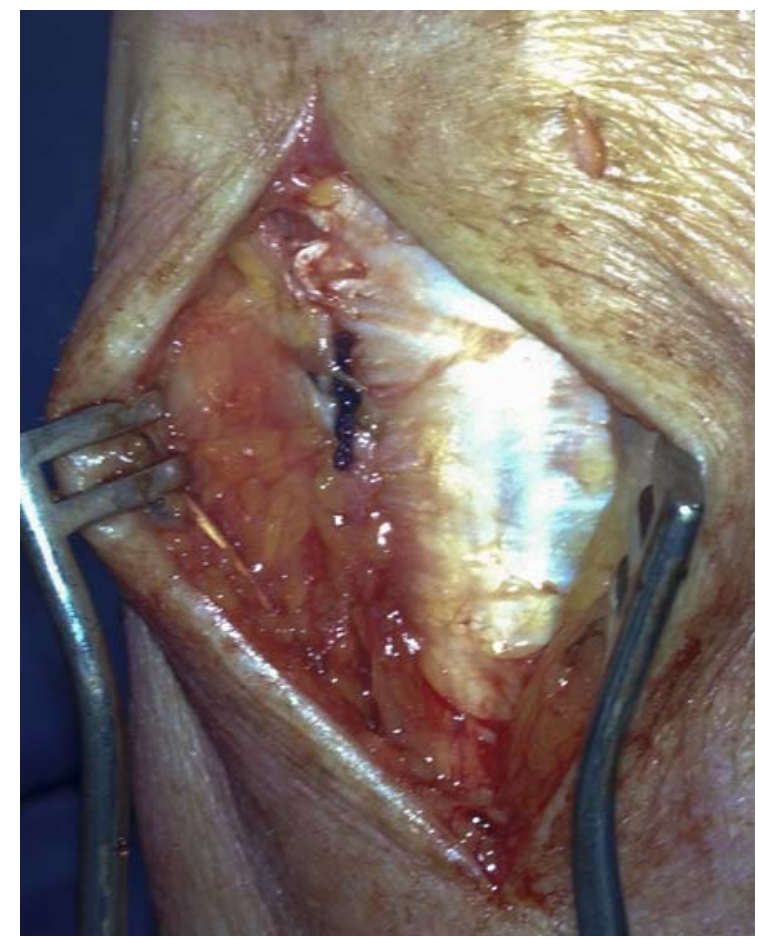

FIGURE 7. The dorsal capsule is plicated which further adds stability to the distal radioulnar joint (DRUJ). $\frac{\text { full color }}{\ln l i n \mathrm{e}}$ 

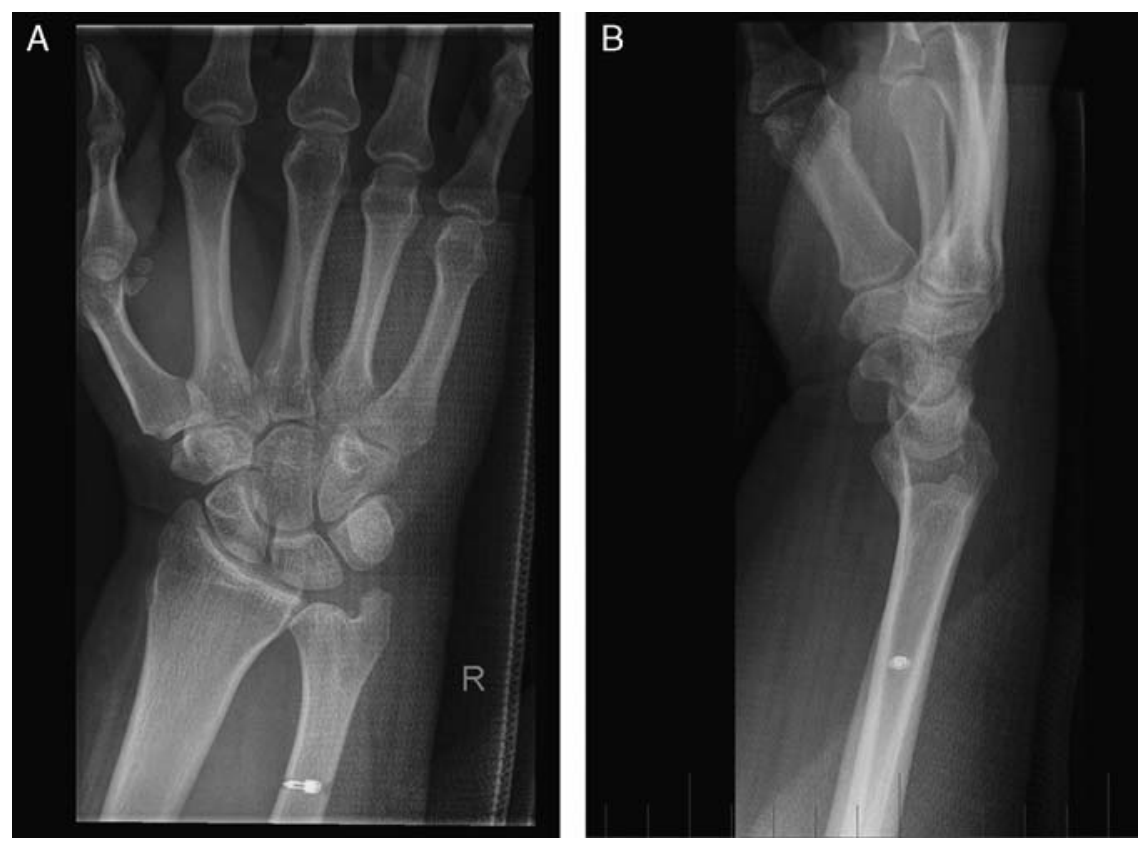

FIGURE 8. A and B, Postoperative radiographs following triangular fibrocartilage complex (TFCC) reconstruction using a palmaris longus tendon graft secured using a tensionable suture anchor in the ulna diaphysis.
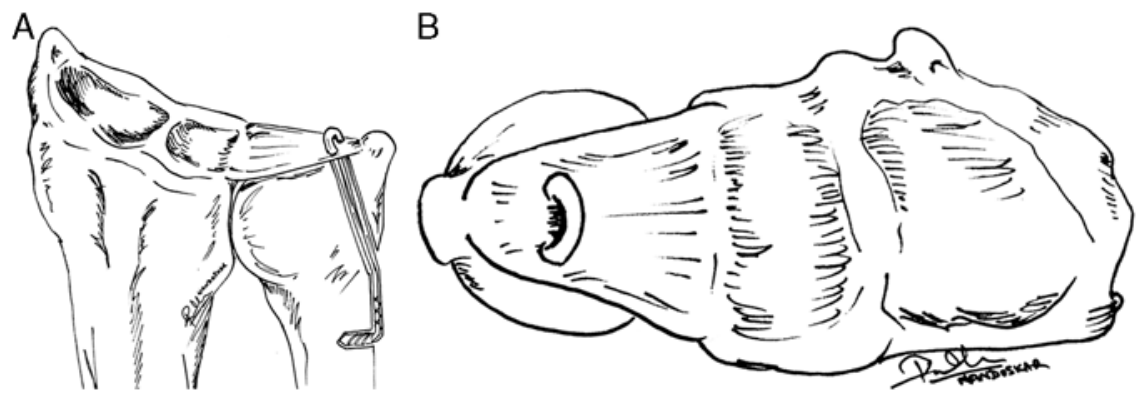

FIGURE 9. A and B, Drawings illustrating our technique of foveal reconstruction of the triangular fibrocartilage complex (TFCC). The palmaris longus tendon is passed through an osseous tunnel in the ulna, weaved through the TFCC and secured proximally to the ulna shaft with a tensionable anchor. Copyright Gregory I. Bain, North Adelaide, Australia. All permission requests for this image should be made to the copyright holder.

A therapist supervises gentle range of motion exercises. At 8 weeks postoperatively, the splint is discarded and full active range of motion and strengthening exercises are encouraged. The patient is advised to not perform any heavy lifting for 3 months.

\section{COMPLICATIONS}

The dorsal cutaneous branch of the ulnar nerve is at risk. There is risk of fracture to the ulnar styloid or distal ulna because of a small bone bridge. It is important to ensure that there is a bone bridge of at least $1.5 \mathrm{~cm}$ from the ulnar styloid to the drill hole, and considerable care is required if an interference fit screw is used to secure the tendon graft. Persistent instability would occur if the tendon graft were to cut through the TFCC; therefore, care is required to ensure the correct placement of the graft through the TFCC and that it is adequately secured.

\section{REFERENCES}

1. Atzei A, Luchetti R. Repair of the foveal insertion of the TFCC through the DF portal. In: Slutsky D, ed. Principles and Practice of Wrist Surgery. Philadelphia: Saunders; 2010:559-568.

2. Atzei A, Luchetti R. Foveal TFCC tear classification and treatment Hand Clin. 2011;27:263-272.

3. Haugstvedt JR, Berger RA, Nakamura T, et al. Relative contributions of the ulnar attachments of the triangular fibrocartilage complex to the dynamic stability of the distal radioulnar joint. J Hand Surg Am. 2006;31:445-451.

4. Moritomo H. Advantages of open repair of a foveal tear of the triangular fibrocartilage complex via a palmar surgical approach. Tech Hand Up Extrem Surg. 2009;13:176-181.

5. Nakamura T, Sato K, Okazaki M, et al. Repair of foveal detachment of the triangular fibrocartilage complex: open and arthroscopic transosseous techniques. Hand Clin. 2011;27:281-290. 
6. Nakamura T, Takayama S, Horiuchi Y, et al. Origins and insertions of the triangular fibrocartilage complex: a histological study. J Hand Surg Br. 2001;26:446-454.

7. Slutsky DJ. Arthroscopic evaluation of the foveal attachment of the triangular fibrocartilage. Hand Clin. 2011;27:255-261.

8. Palmer AK. Triangular fibrocartilage complex lesions: a classification. J Hand Surg Am. 1989;14:594-606.

9. Estrella EP, Hung LK, Ho PC, et al. Arthroscopic repair of triangular fibrocartilage complex tears. Arthroscopy. 2007;23:729-737.

10. Iwasaki N, Nishida K, Motomiya M, et al. Arthroscopic-assisted repair of avulsed triangular fibrocartilage complex to the fovea of the ulnar head: a 2- to 4-year follow-up study. Arthroscopy. 2011; 27:1371-1378.

11. Atzei A. New trends in arthroscopic management of type 1-B TFCC injuries with DRUJ instability. J Hand Surg Eur. 2009;34:582-591.

12. Tay SC, Tomita K, Berger RA. The "ulnar fovea sign" for defining ulnar wrist pain: an analysis of sensitivity and specificity. J Hand Surg Am. 2007;32:438-444.

13. Moriya T, Aoki M, Iba K, et al. Effect of triangular ligament tears on distal radioulnar joint instability and evaluation of three clinical tests: a biomechanical study. J Hand Surg Eur. 2009;34:219-223.
14. Pederzini L, Luchetti R, Soragni O, et al. Evaluation of the triangular fibrocartilage complex tears by arthroscopy, arthrography, and magnetic resonance imaging. Arthroscopy. 1992;8:191-197.

15. Adams BD, Berger RA. An anatomic reconstruction of the distal radioulnar ligaments for posttraumatic distal radioulnar joint instability. J Hand Surg Am. 2002;27:243-251.

16. Mansat M, Mansat C, C Martinez. L'articulation radio-cubitale inferieure. Pathologie traumatique. In: Razemon J, Fisk G, eds. Le Poignet. Paris: Expansion Scientifique Francaise; 1998:196-203.

17. Chun S, Palmer AK. The ulnar impaction syndrome: follow-up of ulnar shortening osteotomy. J Hand Surg Am. 1993;18:46-53.

18. Friedman SL, Palmer AK. The ulnar impaction syndrome. Hand Clin. 1991;7:295-310.

19. Wallwork NA, Bain GI. Sigmoid notch osteoplasty for chronic volar instability of the distal radioulnar joint: A case report. J Hand Surg Am. 2001;26:454-459.

20. Tham SK, Bain GI. Sigmoid notch osseous reconstruction. Tech Hand Up Extrem Surg. 2007;11:93-97.

21. Bain GI, Richards RS, Roth JH. Wrist Arthroscopy. In: Lichtman DM, Alexander AH, eds. The Wrist And Its Disorders. 2nd ed. Philadelphia: WB Sanders; 1997:151-168. 